\title{
STOCHASTIC CLUSTERED-DOT DITHERING
}

\author{
Victor Ostromoukhov ${ }^{1}$, Roger D. Hersch ${ }^{2}$ \\ Ecole Polytechnique Federale, Lausanne (EPFL)
}

\begin{abstract}
A new technique for building stochastic clustered-dot screens is being proposed. A large dither matrix comprising thousands of stochastically laid out screen dots is constructed by first laying out the screen dot centers. Screen dot centers are obtained by placing discrete disks of a chosen radius at free cell locations when traversing the dither array cells according to either a discretely rotated Hilbert space-filling curve or a random space-filling curve. After Delauney triangulation of the screen dot centers, the maximal surface of each screen dot is computed and iso-intensity regions are created. This iso-intensity map is converted into an anti-aliased grayscale image, i.e. into an array of preliminary threshold values. These threshold values are renumbered to obtain the threshold values of the final dither threshold array. By changing the disk radius, the screen dot size can be adapted to the characteristics of particular printing devices. Larger screen dots may improve the tone reproduction of printers having important dot gain.
\end{abstract}

Keywords: Color printing, halftoning, stochastic screening, clustered-dot dithering, space-filling curves

\section{Introduction}

Traditional periodic screening based on different angles and frequencies for the different color layers is by far the most frequently used technique in offset color printing.

Stochastic screening represents an alternative solution for color reproduction (see [Widmer92], [Seybold93], [Ostromoukhov93], [Eschbach94], [Kang96]). It appeared in the mid 1980s and became popular in the early 1990s since it offers finer grain compared to traditional screening. It also permits to superimpose more than three layers without Moirés. This method has shown its usefulness for high resolution devices at relatively high lineatures. However, it requires the printing process to be controlled in order to keep the dot gain within a given range of tolerance.

Today, with the advent of high-resolution desktop color printers (electro-photography, ink-jet) there is a clear need to re-design existing stochastic screening techniques in order to adapt them to these new devices. This is especially the case for electrophotographic color printers which show a non-linear behavior different from the behavior of offset printers. Tuning the size of the printed dot according to the printer's characteristics may considerably improve the tone reproduction curve.

The present article explains the process of building variable-size stochastic screens. We propose methods to build a large size dither matrix which may comprise between 2500 and 10000 stochastically laid out screen dots. In section 2, we describe several methods to lay out the dots within the large dither matrix. Once the screen dots are laid out, i.e. their centers are positioned, we show in section 3 how to create dither threshold levels ensuring a linear dot growth behavior across all gray levels. In section 4 , we draw the conclusions.

\section{Screen dot layout within a large dither matrix}

Regarding the terminology, we use the term screen dot or simply dot for a set of black pixels clustered around a center point. We use the term dither cell or simply cell for a dither matrix element containing one threshold level and being used to generate one pixel of the output image.

1. http://lspwww.epfl.ch/ victor

2. Email: RD.Hersch@epfl.ch

In Color Imaging: Device-Independent Color, Color Hardcopy, and Graphic Arts IV, SPIE Vol. 3648, pp. 496-505, 1999. Reprinted in Journal of Electronic Imaging, Special issue on Color Imaging: Device-Independent Color, Color Hardcopy, and Graphic Arts, October 1999. 
The layout of the dots within a large dither matrix should respect the following requirements:

a) Low frequencies should be avoided, i.e. the most representative frequencies present in the rendered halftone patterns should correspond to the screen dot periods.

b) The frequencies present in the rendered halftone patterns should be isotropic, i.e. there should be no stronger screen frequencies at specific angles.

c) The produced final dither matrix will cover only a part of the final image. It should therefore, when repeated horizontally and vertically, cover the whole image without creating any discontinuities. The final large dither matrix should therefore be continuous across its borders, i.e. the matrix should wrap around, horizontally and vertically.

(a)

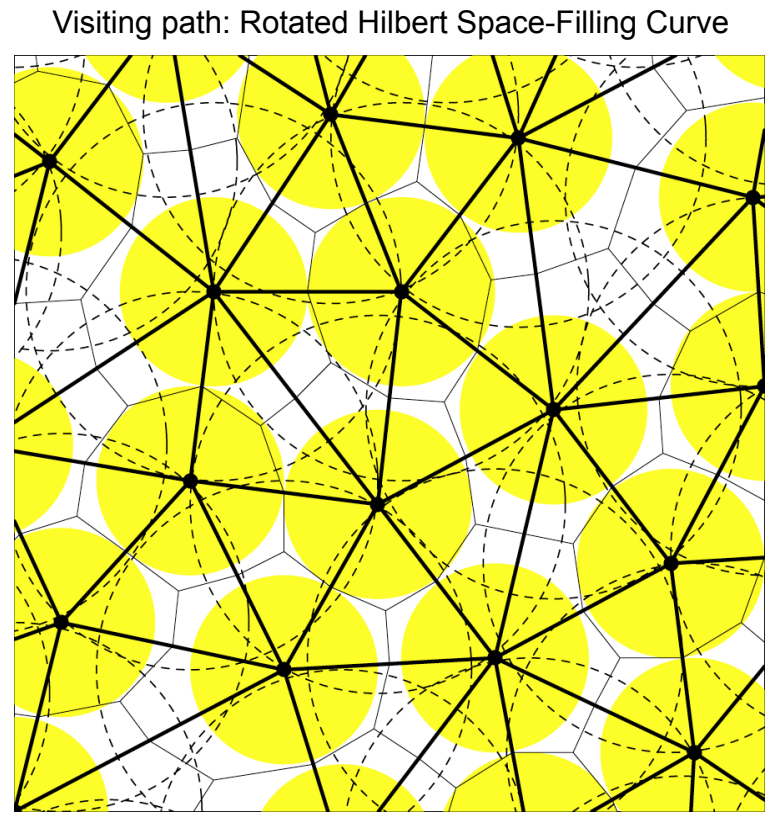

(b)

(c)
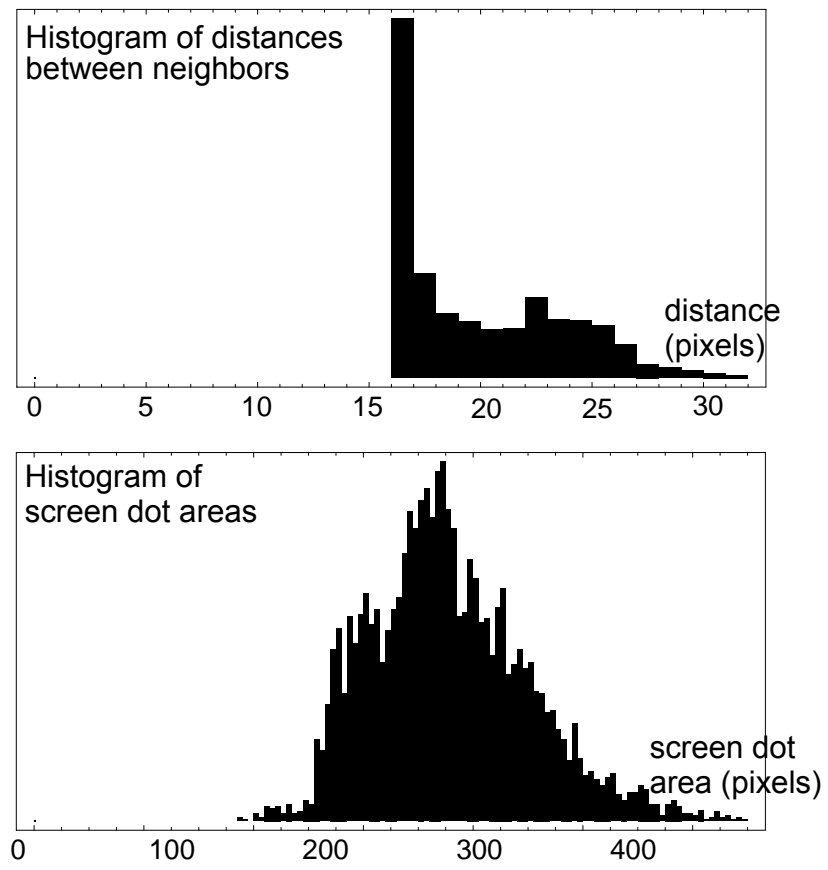

Visiting path: Random Space-Filling Curve
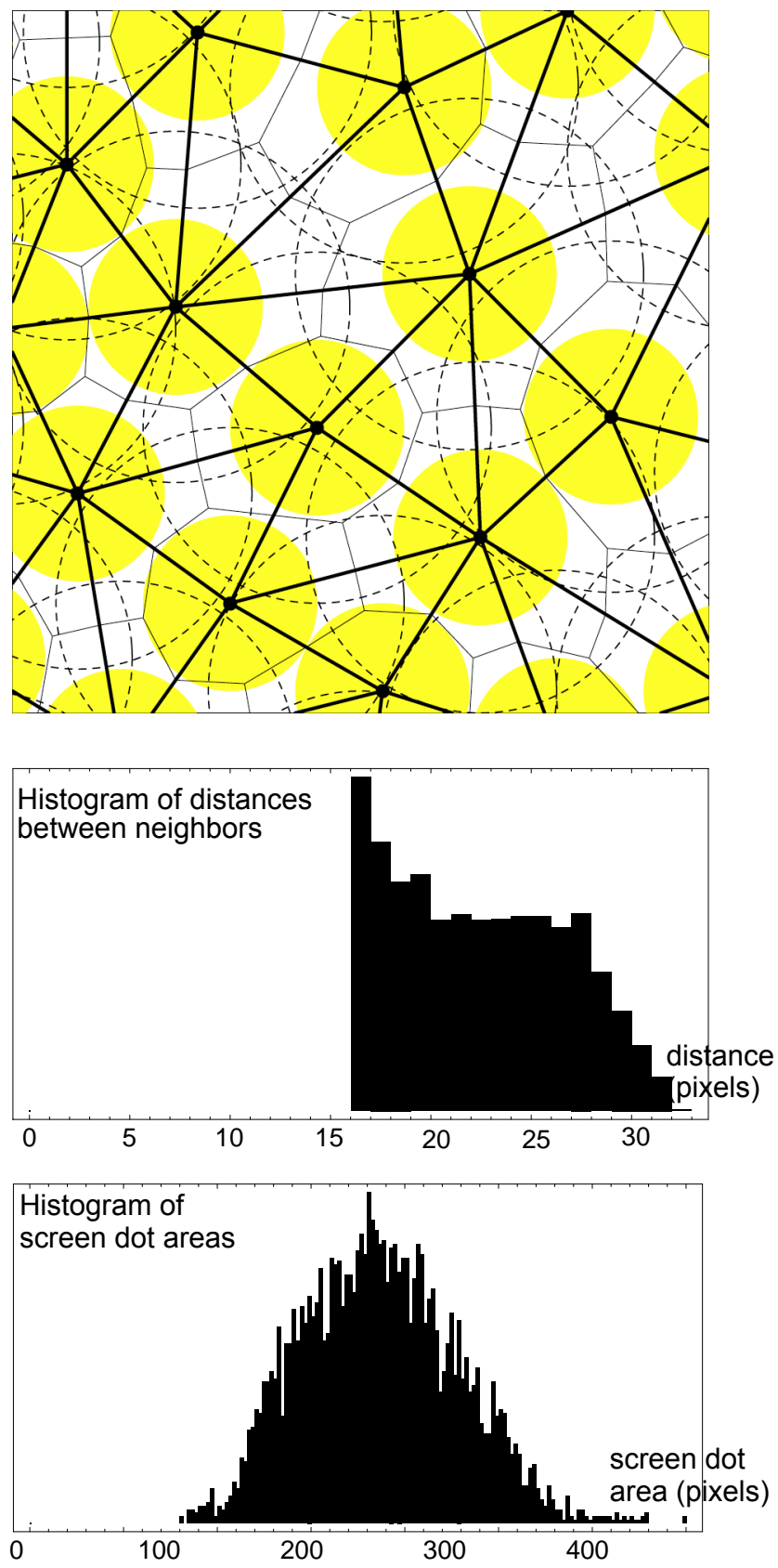

Fig. 1. (a) Matrix covered by partially overlapping disks (dotted circles). The disks shown have a radius of $r / 2$. Thick solid lines show the corresponding Delauney triangulation. Thin solid lines show the limits of the screen dots. (b) Histogram of screen center distances for screen dots generated with a disk radius of 16. (c) Histogram giving the distribution of screen dot surfaces for screen dots generated with a disk radius of 16 . 
To layout the centers of the dots, we can define the minimal distance between two dots as the radius $r$ of a disk. To obtain a spatial distribution of the positions of the centers of all screen dots within the dither matrix, we propose to first mark all cells of the dither matrix as free cells. We then define a visiting path enabling us to visit all cells of the dither matrix. At each visited free cell, we place a discrete disk having a radius of $r$ cells. All cells covered by such a disk are marked as occupied. Since the visiting path traverses all matrix cells, the whole matrix becomes covered by partially overlapping disks (dotted circles in Fig. 1).
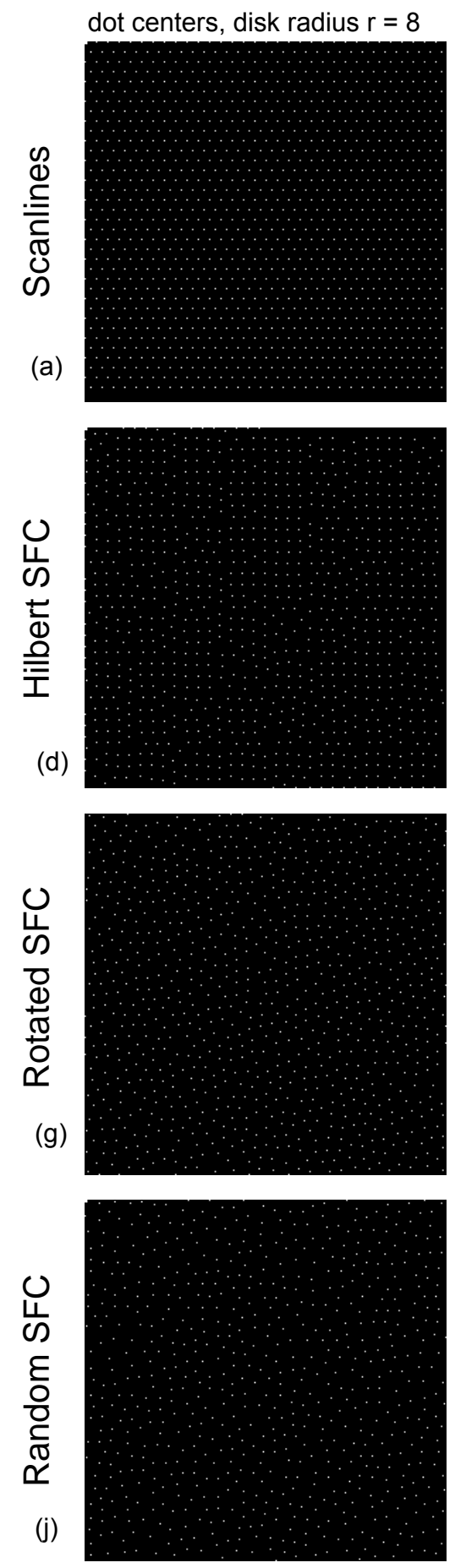

dot centers, disk radius $r=16$

(b)

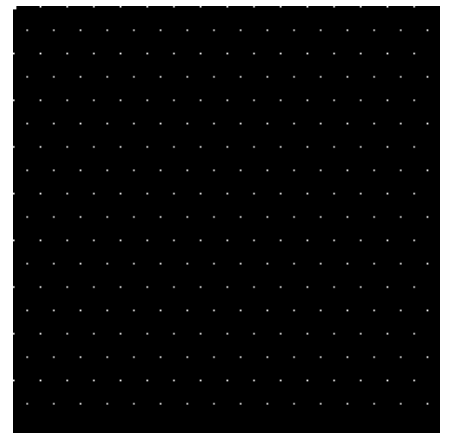

(e)

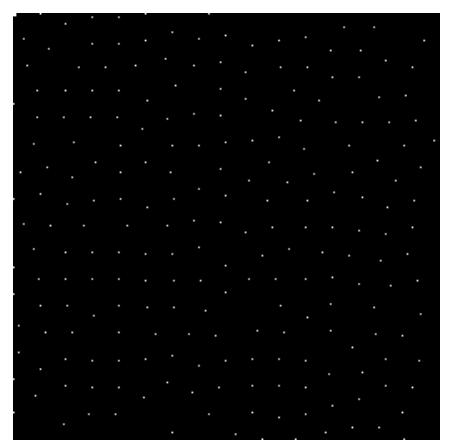

(h)

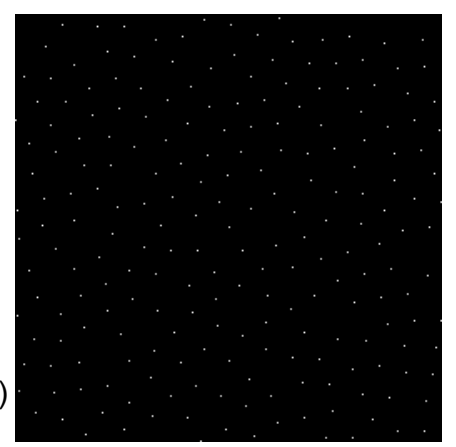

(k)

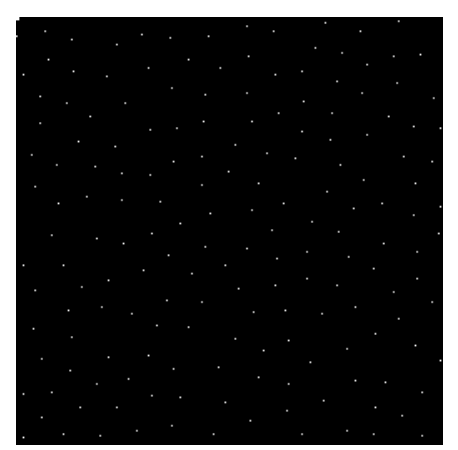

Fourier amplitude spectrum of dot center images, $r=16$

(c)

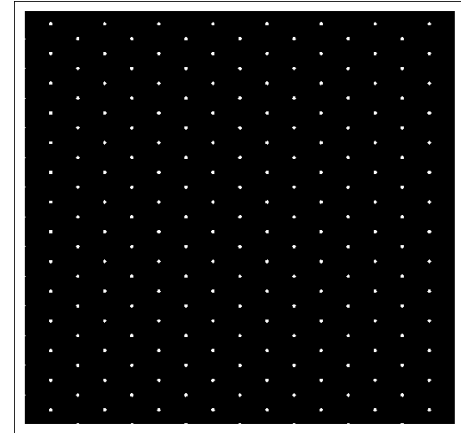

(f)

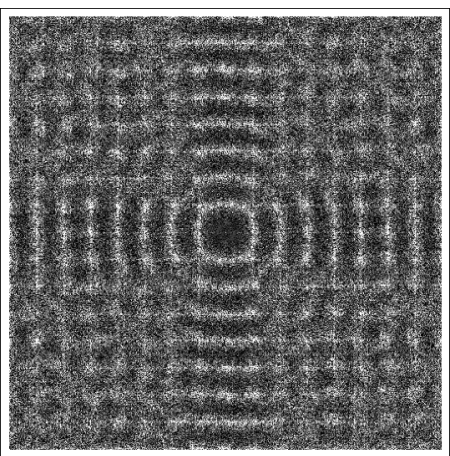

(i)

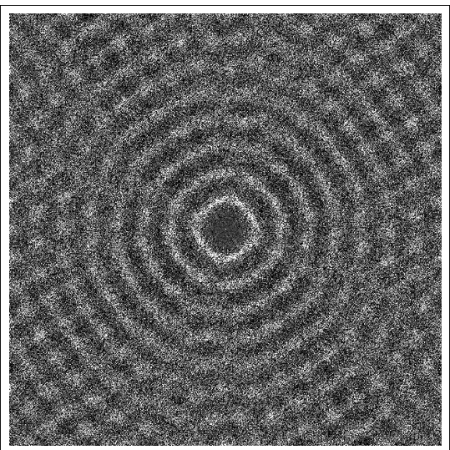

(I)

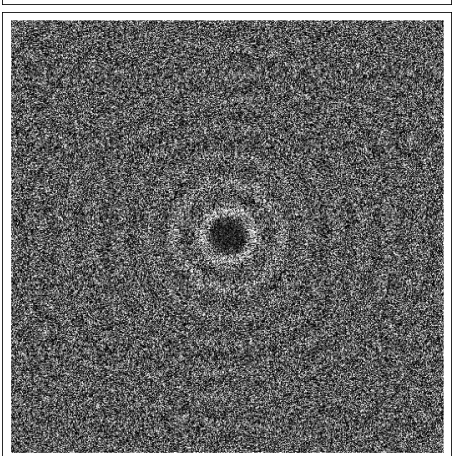

Fig. 2. Dot centers produced by visiting the dither array (a), (b) scanline by scanline, (d), (e) according to the Hilbert spacefilling curve, (g), (h) according to the rotated Hilbert space-filling curve, (j), (k) according to a random space-filling curve and (c), (f), (i), (l) the corresponding Fourier Amplitude spectra. 
All disk centers become the center of a screen dot. To define the maximal surface of each screen dot, a Delauney triangulation is applied to the set of centers [Goodman97]. The corresponding Voronoi diagram may have been used to define the maximal surface coverage of each stochastically laid out screen dot. However, to obtain well clustered black dots at high intensity levels and well clustered white dots at low intensity levels, an equivalent, but slightly different scheme is used to create the maximal surface coverage of each screen dot (see section 3 ).

\subsection{Visiting path: scanline by scanline}

The properties of the dot distribution will be determined by the visiting path. A naive visiting path scanning the dither matrix cell by cell and scanline by scanline produces a set of periodically laid out and tightly packed screen dot centers (Fig. 2a and 2b). The resulting screen is a periodic diagonally oriented screen. The basic screen frequencies are located at particular locations and are therefore not isotropic.

\subsection{Visiting path: Hilbert space-filling curve}

To improve the dot distribution, we can use as a visiting path the Hilbert space-filling curve [Sagan94]. In that case, the dither matrix is of size N, where N is a power of two. Since parts of the Hilbert space-filling curve incorporate some symmetry properties, the resulting dot center distribution is again isotropic (Fig. 2d-f).

\subsection{Visiting path: rotated Hilbert space-filling curve}

An improved distribution of dot centers is obtained by creating a visiting path made of a Hilbert space-filling curve rotated by a one-to-one discrete rotation[Ostromoukhov94]. The rotated space-filling path is obtained by rotating a multiple of the original Hilbert space-filling path with a Pythagorean angle $\operatorname{atan}(3 / 4)$.

Such a discrete one-to-one rotation is obtained by rotating with a Pythagorean angle $\alpha=\operatorname{atan}(3 / 4)=36.87^{\circ}$ and by applying rounding operations. Let us assume that $(u, v)$ are the coordinates of the original dither array cells (the lower-left cell lies at the origin) and $(x, y)$ the coordinates of the rotated dither array cells. Then the discrete one-toone rotation is expressed by:

$$
\begin{aligned}
& x=\operatorname{round}\left(\frac{a}{c} \cdot u-\frac{b}{c} \cdot v\right) \\
& y=\operatorname{round}\left(\frac{b}{c} \cdot u+\frac{a}{c} \cdot v\right)
\end{aligned}
$$

where $c=5, a=4$ and $b=3$.

To be horizontally and vertically repetitive, any structure such as a matrix or a discrete path to be rotated by a Pythagorean angle $\alpha=\operatorname{atan}(3 / 4)$ must have a horizontal and vertical period which is a multiple of 5 [Ostromoukhov94]. Therefore, one must choose to rotate a dither array made of a single Hilbert curve, of size $5 \mathrm{~N}$ by $5 \mathrm{~N}$, where $\mathrm{N}$ by $\mathrm{N}$ is the size of the array containing the Hilbert curve. Fig 3 shows the original $4 \times 4$ Hilbert space-filling path and a rotated space-filling path obtained by rotating a multiple of the original Hilbert space-filling path by the Pythagorean angle $\alpha=\operatorname{atan}(3 / 4)=36.87^{\circ}$.

The rotated Hilbert space-filling curve is asymmetric. One can observe both in the image space (Fig. $2 \mathrm{~g}$ and $2 \mathrm{~h}$ ) and in the Fourier amplitude spectrum (Fig. 2i) that the resulting dot center distribution is almost isotropic.

\subsection{Visiting path: Random space-filling curve}

Let us explore the results obtained by a random space-filling curve. A random space-filling curve where proximity of successive cells is not required can be constructed by starting from a list of cells, initially ordered cell by cell and scanline by scanline. Two cells within this ordered list are randomly selected and permuted. After a number of permutations, the cell visiting order is completely randomized. There is absolutely no symmetry in the sequence of visited cells. As expected, one can observe both in the image space (Fig. $2 \mathrm{j}$ and $2 \mathrm{k}$ ) and in the Fourier amplitude spectrum (Fig. 2l) that the resulting dot center distribution is isotropic. Furthermore, multiples of the basic frequency components are much less pronounced than in the case of the rotated Hilbert space-filling curve visiting path.

Methods for finding screen dot layout presented in this article differ from other methods known in the art (see for example [Eschbach90], [Marcu98]). 
(a)
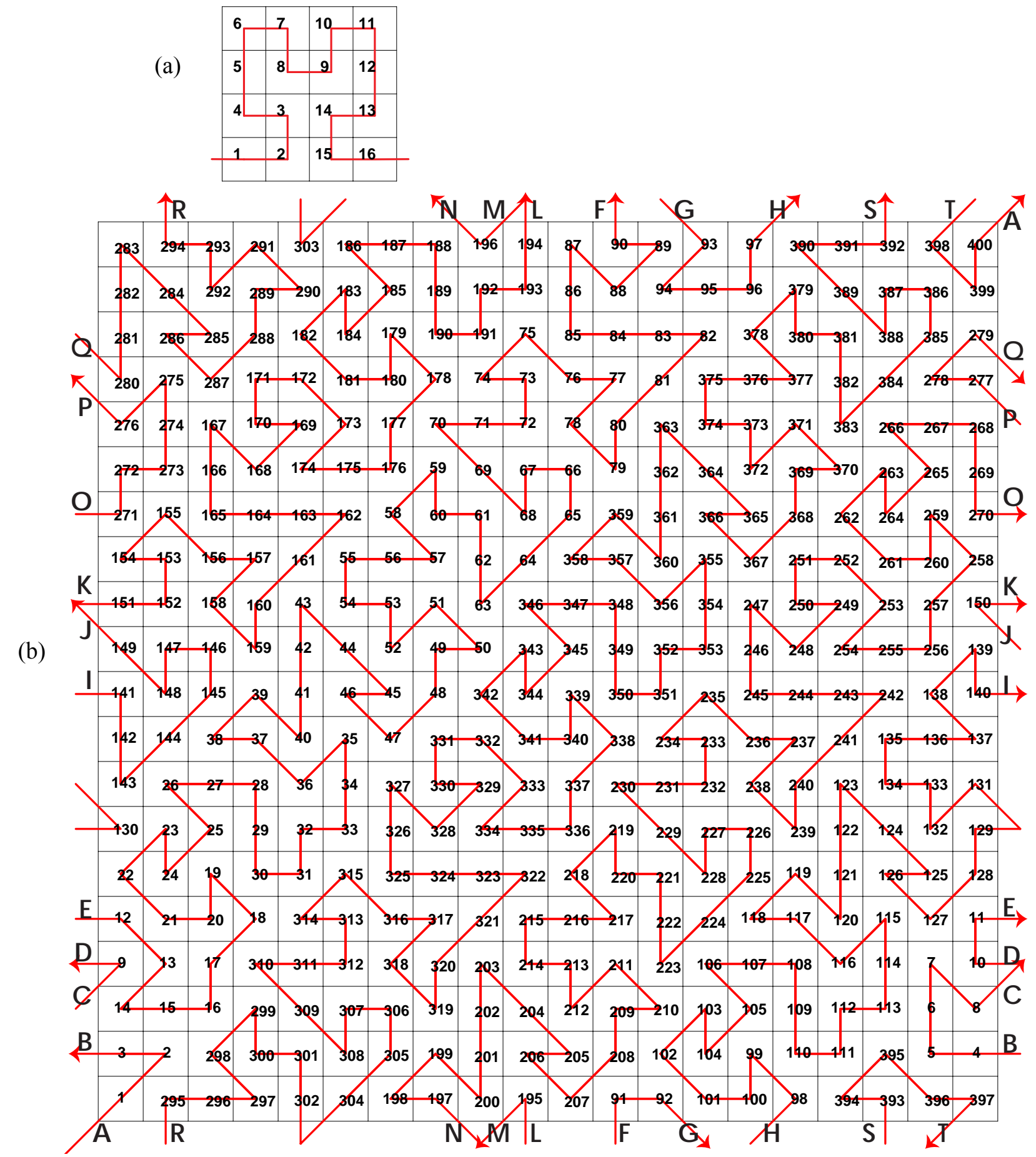

Fig 3. (a) Original $4 \times 4$ Hilbert space-filling path and (b) a rotated space-filling path obtained by rotating a multiple of the original Hilbert space-filling path by the Pythagorean angle atan(3/4).

\section{Generating the dither matrix with stochastic screen dots}

Starting from a set of stochastically distributed screen dot centers, the final dither matrix is obtained with the following steps:

a) Generate a Delauney triangulation of the set of screen dot centers

b) From the Delauney triangulation, define the full surface coverage of each screen dot (maximal surface coverage)

c) Generate iso-intensity lines around each screen dot for a set of different intensity levels (for example for 32 
intensity levels)

d) With a standard software package, such as Photoshop, according to the defined iso-intensity lines, create threshold levels for all cells of the dither matrix (this corresponds to the generation of a grayscale image according to the given iso-intensity lines)

e) Renumber the previously generated threshold levels so as to ensure a uniform distribution of threshold levels, i.e. a flat histogram of the dither threshold levels present in the dither matrix.

Many books describe the Delauney triangulation of a planar set of dots (see [Goodman97]). Once the triangulation has been carried out, the full surface of each screen dot is determined geometrically by connecting triangle centers with their respective edge middle points (Fig. 4a).

Iso-intensity lines are determined so as to ensure that the surface within two successive iso-intensity lines grows linearly with intensity. Therefore, the positions of successive intersections of iso-intensity lines and triangle boundaries needs to be appropriately computed, taking into account that the quotient of the surfaces of two scaled triangles is the square of the quotient of their respective side lengths. Figure $4 \mathrm{c}$ illustrates the generated iso-intensity lines defining equally large surfaces for a certain numbers of screen dots.

(a)
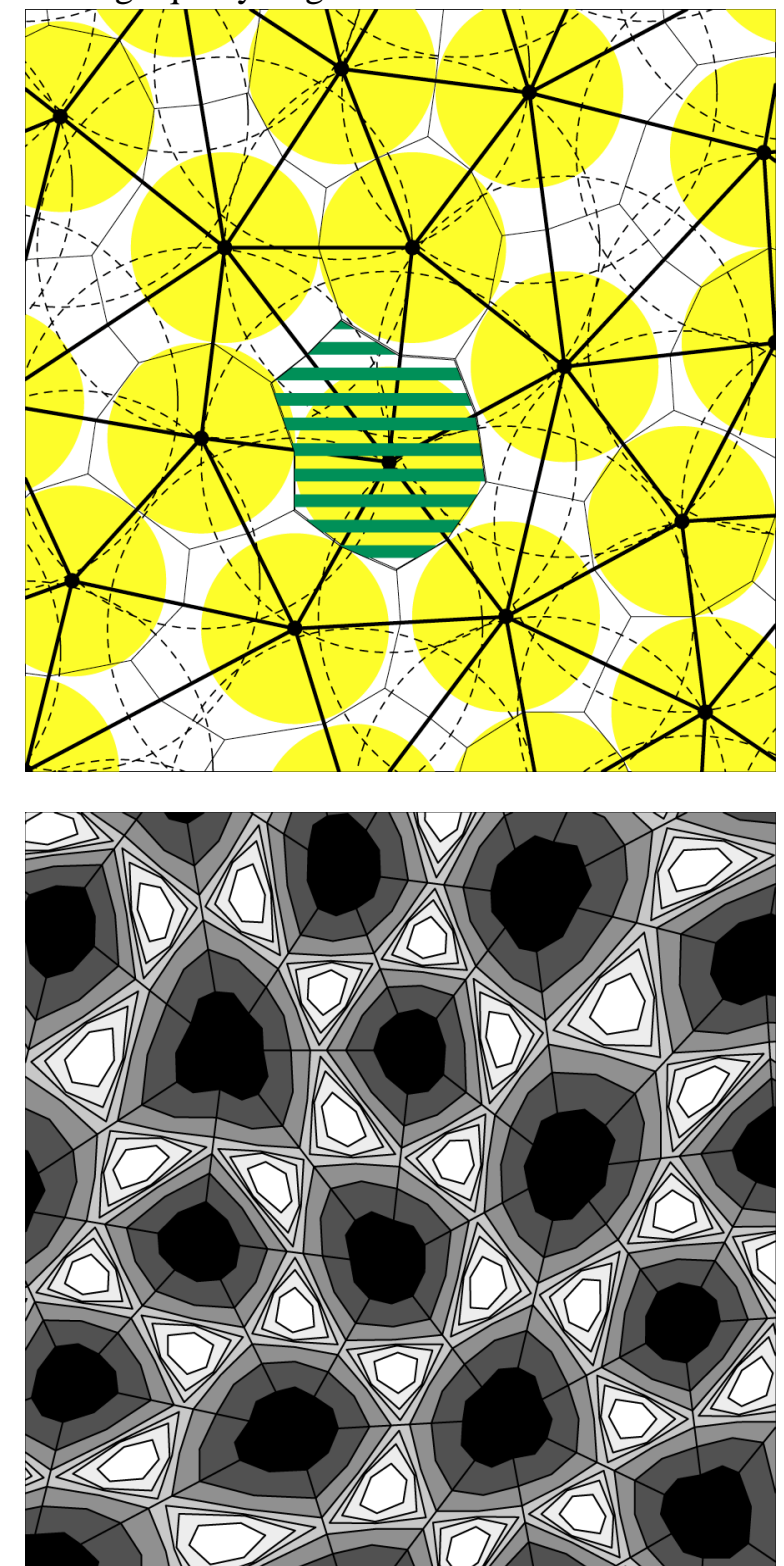

(b)

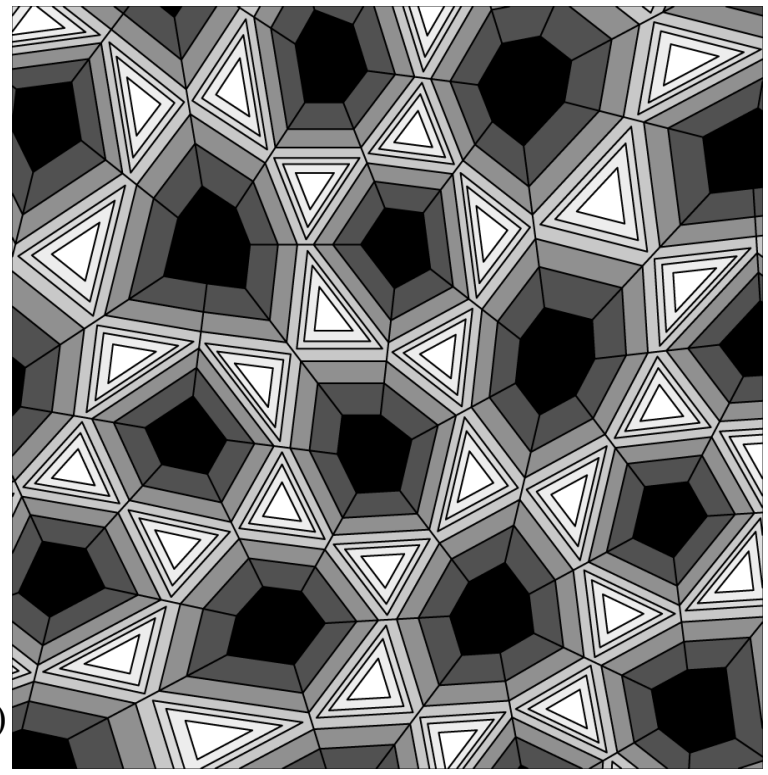

(d)

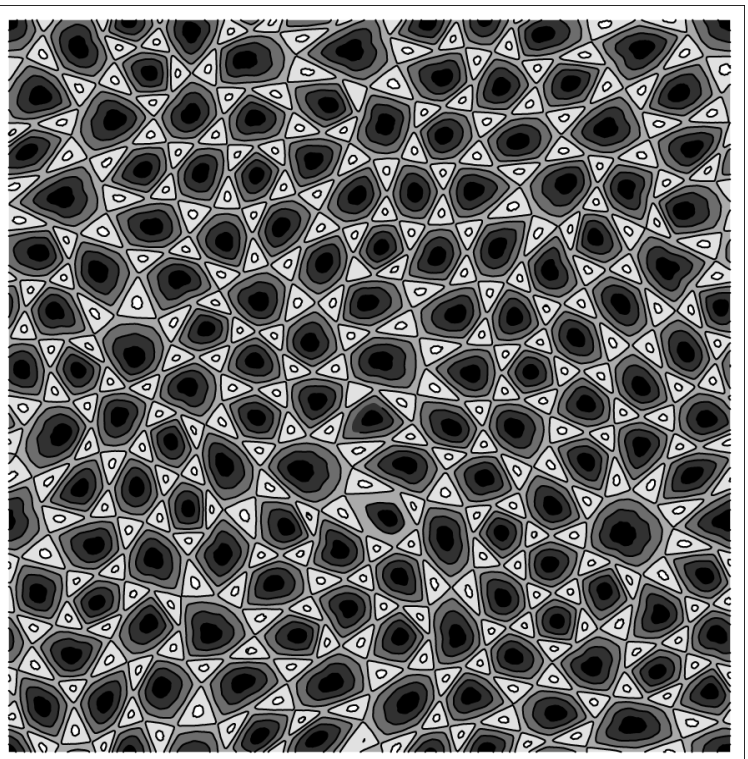

Fig. 4. Creation of a stochastic dither matrix using a rotated Hilbert visiting path: (a) maximal screen dot surface (hatched); (b) creation of iso-intensity lines by subdividing the triangles' edges into appropriate intervals; (c) modified iso-intensity lines; and (d) detail of the resulting dither matrix, plotted with a few discrete intensity levels bounded by iso-intensity curves. 
Because of the subsequent renumbering of cells, the iso-intensity lines are allowed to incorporate some variations and the generated surfaces are allowed to differ slightly one from another. Fig. 4d shows the iso-intensity lines generated so as to produce screen dots with additional vertices, i.e. smoother screen dots than the screen dots produced in figure 4c.

The generated iso-intensity line artwork is rasterized into an anti-aliased grayscale image using a standard imaging software package (for example Adobe PhotoShop). The grayscale levels are an approximation of the desired threshold levels and represent a surface interpolation of the previously generated iso-intensity lines.

These grayscale levels are renumbered, i.e. identical gray levels are assigned successive numbers. The resulting renumbered array is the desired dither threshold array: the renumbering process ensures that dither threshold levels are uniformly distributed.

The generated large size dither array is used to create halftones with a stochastic screen dot. The disk radius $r$ defines the minimal distance between the screen dots. The histogram of screen dot center distances shows that screen dots are apart by a maximal distance of $2 r$ (Fig. 1b). Histogram 1c gives a histogram of the surface associated to screen dots, for a disk radius of 16 pixels. It shows that the minimal screen dot surface is close to that of a disk with radius $r / 2$.

Figure $4 \mathrm{~b}$ shows the dither matrix produced by the random space-filling visiting path. To provide a sharper view of the screen dots, the dither matrix is plotted with a few discrete intensity levels bounded by iso-intensity curves.

Fig 5a shows a wedge rendered with the dither matrix shown in Fig. 4b (disk radius $r=16$ ), and Fig 5b shows a wedge rendered with a dither matrix generated using a disk of radius $r=8$. Observe the generated stochastic screen dots: there is no predominant orientation and no low frequency patterns are visible.

(a)

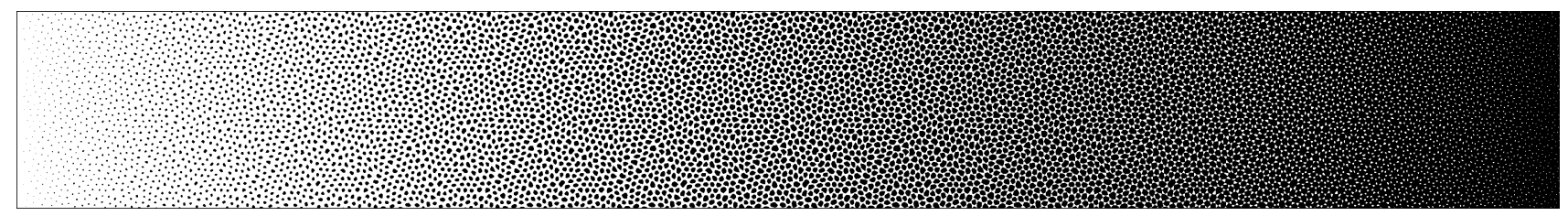

(b)

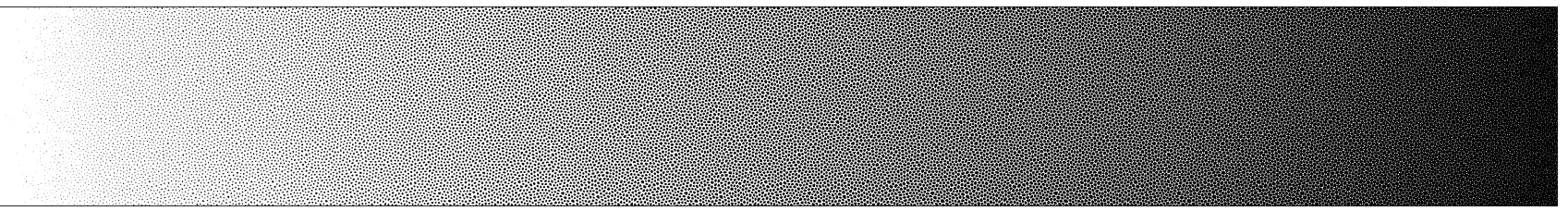

Fig 5. (a) Grayscale wedge rendered with the dither matrix shown in Fig. 4b, using the rotated Hilbert space-filling curve, disk radius $r=16$, printed at 600 dpi. (b) Grayscale wedge rendered with a dither matrix using the rotated Hilbert space-filling curve, disk radius $\mathrm{r}=8$, printed at $600 \mathrm{dpi}$.

Please note the non-symmetric behavior of the screen dots in highlight and shadow regions. According to the layout of the screen dots (Fig. 4b), the number of white dots is approximatively twice the number of black dots. Therefore, in highlight regions, black dots have a surface which is twice as large as white dots in corresponding shadow regions.

\section{Conclusions}

A new technique for building stochastic clustered-dot screens has been described. A large dither matrix comprising thousands of stochastically laid out screen dots is constructed by first laying out the screen dot centers. Screen dot centers are obtained by placing discrete disks of the chosen radius $r$ at free cell locations when traversing the dither array cells according to either a discretely rotated Hilbert space-filling curve or a random space-filling curve. After Delauney triangulation of the screen dot centers, the maximal surface of each screen dot is computed and iso-intensity regions are created. This iso-intensity map is converted into an anti-aliased grayscale image, i.e. into an array of preliminary threshold values. These threshold values are renumbered to obtain the threshold values of the final dither threshold array.

The resulting stochastic clustered dot screen incorporates screen dots whose distances vary between the chosen disk radius $r$ and $2 r$. For the random space-filling curve, the histogram of the screen dot surface is similar to a 
Gaussian function. The largest screen dot surface is roughly 3 times larger than the smallest screen dot surface. By changing the disk radius, the screen dot size can be adapted to the characteristics of particular printing devices. Larger screen dots may improve the tone reproduction of printers having important dot gain.

The stochastic screens produced using the presented method look like fine mezzotint (see Figs 6 and 7). The proposed stochastic screen generation method is of interest for high-resolution office printers: at $1200 \mathrm{dpi}$, a stochastic screen generated with a disk of radius $r=8$ corresponds to a traditional screen of 150 lpi. The extension of the presented method to color images is underway. This extension will also incorporate sophisticated dot gain compensation methods.

\section{References}

[Eschbach90] Reiner Eschbach, Pulse-density modulation on rastered media: combining pulse-density modulation and error diffusion, $J O S A(A)$, Vol. 7, No. 4, pp. 708-716, 1990.

[Eschbach94] Reiner Eschbach (Ed.), Recent Progress in Digital Halftoning, IS\&T, 1994.

[Goodman97] Jacob E. Goodman (Ed.), Joseph O'Rourke, Jacob Joseph, Handbook of Discrete and Computational Geometry (CRC Press Series on Discrete Mathematics and Its Applications), CRC Press, 1997.

[Kang96] Henry R. Kang, Color Technology for Electronic Imaging Devices. SPIE Optical Engineering Press, 1996.

[Marcu98] Gabriel Marcu, An error diffusion algorithm with output position constraints for homogenious highlights and shadow dot distribution, In Color Imaging: Device-Independent Color, Color Hardcopy, and Graphic Arts III, SPIE Vol. 3300, pp. 341-352, 1998.

[Ostromoukhov93] V. Ostromoukhov, Pseudo-Random Halftone Screening for Color and Black\&White Printing. Proceedings of the 9th Congress on Advances in Non-Impact Printing Technologies, Yokohama, pp. 579-581, 1993.

[Ostromoukhov94] V. Ostromoukhov, R.D. Hersch, I. Amidror, Rotated Dispersed Dither: a New Technique for Digital Halftoning, Proceedings of SIGGRAPH'94, In ACM Computer Graphics, Annual Conference Series, pp. 123-130, 1994.

[Sagan94] Hans Sagan, Space-Filling Curves (Universitext), Springer Verlag, 1994.

[Seybold93] Frequency-Modulated Screening Technology. Seybold, Special Report. Vol. 1, No. 2, 1993.

[Widmer92] Widmer, Erwin, K. Schlaepfer, V. Humbel \& S. Persiev, The Benefits of Frequency Modulation Screening, Technical Association of the Graphics Arts, Vol.1. pp. 28-43, 1992. 


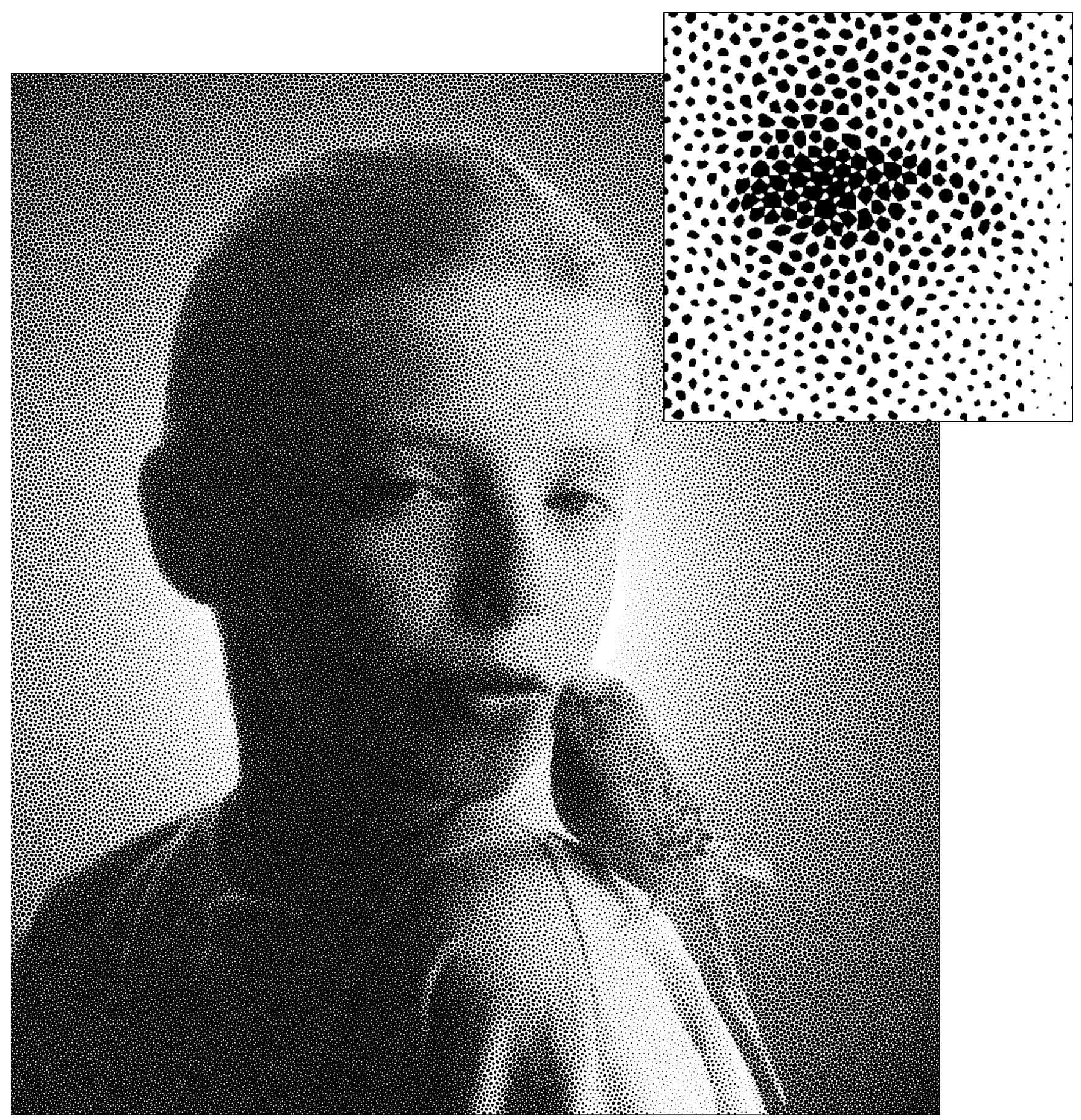

Fig. 6. A sample image, generated with a dither matrix incorporating stochastic screen dots produced with a disk of radius 16 (random space-filling curve), printed at 600 dpi. 


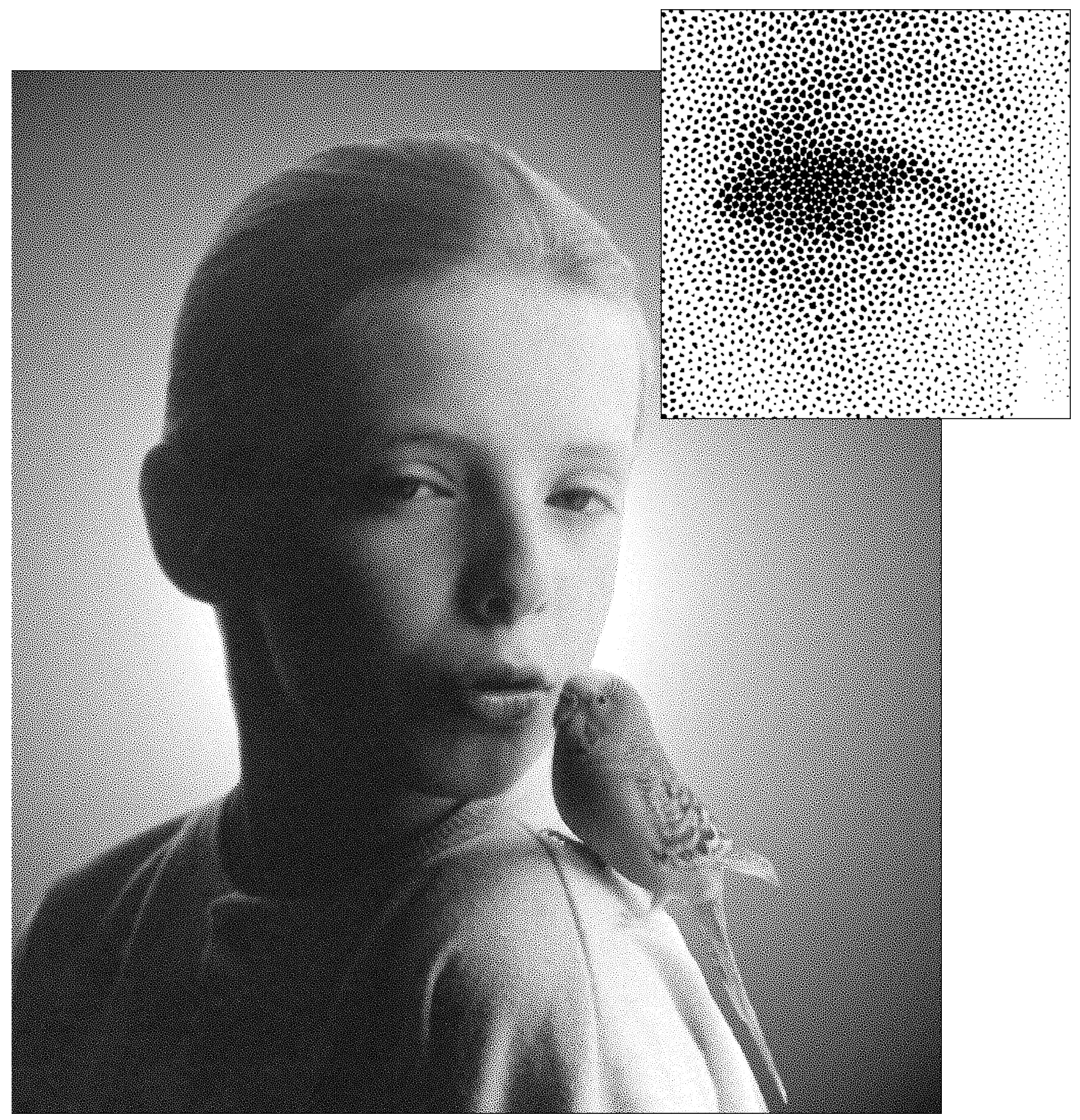

Fig. 7. A sample image, generated with a dither matrix incorporating stochastic screen dots produced with a disk of radius 8 (random space-filling curve), printed at $600 \mathrm{dpi}$. 